\title{
Expert consensus on the prevention and treatment of substance use and addictive behaviour-related disorders during the COVID-19 pandemic
}

Jiang Du, ${ }^{1} \mathrm{Ni}$ Fan, ${ }^{2}$ Min Zhao (D) ${ }^{3}$ Wei Hao, ${ }^{4}$ Tieqiao Liu, ${ }^{5}$ Lin Lu, ${ }^{6}$ Jie Shi, ${ }^{7}$ Haifeng Jiang, ${ }^{1} \mathrm{Na}$ Zhong, ${ }^{1}$ Xiaochang Lan, ${ }^{2}$ Shichao Xu, ${ }^{2}$ Hongxian Chen, ${ }^{5}$ Xiaojun Xiang, ${ }^{5}$ Xuyi Wang, ${ }^{5}$ Hongqiang Sun, ${ }^{6}$ Bing Li, ${ }^{6}$ Yu-Ping Ning, ${ }^{8}$ Jing Li, ${ }^{9}$ Wanjun Guo, ${ }^{9}$ Yajuan Niu, ${ }^{10}$ Lixia Sheng, ${ }^{11}$ Yi Li, ${ }^{12}$ Xuebin Liu, ${ }^{12}$ Xuhui Zhou, ${ }^{13}$ Mincai Qian, ${ }^{14}$ Wenhua Zhou, ${ }^{15}$ Ruiling Zhang, ${ }^{16}$ Hongxing Hu, ${ }^{17}$ Yan Xia, ${ }^{18}$ Zhonghua Su, ${ }^{19}$ Ruimin Zhang, ${ }^{20}$ Mei Yang, ${ }^{21}$ Fen Liu, ${ }^{21}$ Wei Yuan ${ }^{22}$

In early 2020, the COVID-19 outbreak complicated the diagnosis, treatment and rehabilitation of patients with substance use disorders and increased the risks of substance abuse and addictive behaviours, such as online gaming disorders, in the general public. Substance use disorder is a chronic recurrent brain disease characterised by strong cravings, high recurrence rates, and a high proportion of comorbidity of mental and physical disorders. ${ }^{1}$ Therefore, regular long-term therapeutic interventions are critical to preventing drug relapses while maintaining withdrawal.

COVID-19 severely affects normal medical diagnoses, treatments and managements of patients with substance use disorder coupled with addiction-related behaviour. It also adversely impacts the mental state of the general public in several ways and leads to broader symptoms, including anxiety, tension and insomnia, that may increase the risk of alcohol abuse, sedative and hypnotic drug abuse, and other addictive behaviours.

The State Council's Comprehensive Team for Joint Prevention and Control of COVID-19 released a series of documents to address the importance of focusing on the prevention and control of both imported cases and internal transmission in particularly vulnerable places, such as supervision

For numbered affiliations see end of article.

Correspondence to Dr Min Zhao;

drminzhao@smhc.org.cn

Professor Wei Hao; weiha057@163.com sites, pension facilities, welfare homes and mental health institutions. ${ }^{23}$ It is also of utmost importance to ensure the continuous, regular and effective treatment of existing patients with addiction problems, reduce the risk of drug relapse and prevent new cases of addiction during the pandemic.

To this end, the Chinese Association of Drug Abuse Prevention and Treatment, the Academic Group of Drug Dependence of the Chinese Society of Psychiatry, the Academic Group of Addiction of the Chinese Society of Psychosomatic Medicine, and the Specialty Committee of Addiction Medicine of the Chinese Psychiatrist Association jointly published an expert consensus to inform the public with proper guidance to prevent addiction and provide professional diagnoses, treatment and management of these addictions. Since these issues are faced by various groups of people, we addressed these problems based on different populations.

PRACTICAL DIFFICULTIES FACED BY THE GENERAL POPULATION AND PATIENTS WITH ADDICTION PROBLEMS DURING THE PANDEMIC

COVID-19 has caused a serious and comprehensive impact on the public. The dual stress fomented by 'confronting a pandemic' and 'isolation at home' negatively impacts mental health to various degrees. Those affected often manifest symptoms such as tension, worry, fear, depression and distress. If not properly vented, these emotional problems can increase the risk of substance abuse and gaming disorders. ${ }^{4}$ Studies have shown that when stressed, abstinent patients with substance use disorder are more likely to have negative emotions and are preconditioned to return to previous behavioural patterns as a coping mechanism. For example, they relieve anxiety, depression or insomnia through excessive use of tobacco, alcohol or even drugs, all of which lead to relapses or exacerbations of existing conditions. ${ }^{5-7}$

For current patients with addiction problems, the amount and frequency of substance use are amplified when they encounter stressful situations. Therefore, the occurrence, development, addiction relapse and the state of stress may mutually influence and aggravate each other during the pandemic. This in turn increases the aggregated risk. In addition to mutual influences, daily medical treatments for patients with addiction problems are also affected to varying degrees based on restrictive isolation policies and delivery inconveniences during a pandemic. For instance, patients who require methadone maintenance therapy may not be able to receive methadone in a timely manner, and this may increase the risk of psychobehavioural problems and potential relapse.

\section{ADDICTION PROBLEMS AND PREVENTION ADVICE THAT THE GENERAL PUBLIC SHOULD FOCUS ON DURING THE PANDEMIC}

The COVID-19 pandemic is a serious public crisis that has interactive susceptibility with addictive behaviours. Stressful circumstances can increase the risk of addictive behaviour; therefore, it is important to prevent the occurrence of substance use and other addictive behaviours during difficult situations, such as this pandemic.

\section{Addiction problems}

As mentioned, during the pandemicespecially during long periods of isolation-several kinds of stress may lead to negative emotions and related maladaptive coping styles. Among the types of addictive substances and 
behaviours that warrant special attention are abuse of alcohol or sedative hypnotics and compulsive internet gaming.

\section{Alcohol abuse}

As a central nervous system depressant, alcohol is widely used by the general public. In addition to social drinking, many individuals imbibe as a form of self-treatment, such as relief for anxiety or depression, or to help them sleep. However, long-term excessive drinking often leads to alcohol abuse or dependence and can cause a series of physical and mental health problems. During any pandemic, there is an increased risk of fomenting disturbing feelings or aggravating existing negative emotions in patients with addiction problems as well as in the general public. Some will attempt to confront their problems by drinking. This in turn increases the risk of alcohol abuse or eventual addiction. In addition, if alcohol-dependent patients suddenly stop drinking, they may experience severe withdrawal symptoms which could be lifethreatening, such as delirium tremens, epileptic seizures and electrolyte imbalance. Therefore, at-risk persons should avoid drinking as a means to cope with emotional problems or insomnia, and patients with alcohol dependence must be encouraged to remain abstinent when isolated at home.

\section{Sedative hypnotic abuse}

There are many types of sedative and hypnotic drugs, most of which include antianxiety remedies that can mitigate negative emotions caused by tension, anxiety and fear. Sedative hypnotics offer mild and generally positive sedative effects when taken in small dosages. Larger dosages tend to induce sleep. As a pandemic spreads, the number of individuals with anxiety and insomnia likewise increases gradually, as do the risks and potential addictions. Some also use sedatives and hypnotic drugs combined with alcohol to accentuate the effects, and this often results in more serious negative consequences. Therefore, the preferred recommendations for relieving anxiety and insomnia are non-pharmaceutical therapies such as relaxation practices, t'ai chi and yoga. If pharmacotherapy is necessary for acute cases, sedative and hypnotic drugs should only be used under the guidance of professional doctors rather than self-administration.

\section{Overuse of games and the internet}

Gaming disorder is primarily found in adolescents, while excessive internet use is seen in all ages. ${ }^{89}$ With the popularity of the internet and smartphones, there are increased risks of addiction to internet games, short videos, online graphic novels and comics, and even internet gambling, online pornography and other disruptive content. For teenagers, the influencing factors generally include changes to their daily routines and home studies during the pandemic period. Some of the most prevalent characteristics of adolescents are impulsiveness and curiosity. For young and middle-aged adults, the main factors are disruptive changes to their living and working spaces, as well as interruptions to their daily social activities. The elderly are adversely affected by changes to their daily rhythm. Interventions to prevent internet and gaming addictions should be tailored to the specific characteristics of each age group, including regular rest and alternative forms of enriching one's daily life by developing healthy interests and hobbies.

\section{Prevention and suggestions}

During the COVID-19 pandemic, related work can be conducted in accordance with the different risk levels.

\section{General population}

The general public should plan their lives, maintain healthy lifestyles and conform to proper daily routines during isolation periods. Regular sleeping and eating combined with moderate exercise provide individuals with sufficient energy to manage the daily requirements of work and life. Maintain communication with friends and distant family members through email, telephone and social apps such as WeChat. ${ }^{10}$ Those who are physically and mentally capable should make full use of available resources to learn simple, practical and evidence-based self-adjustment methods (eg, muscle relaxation training, mindfulness, negative emotion recognition and coping mechanisms). Harmful coping strategies should be avoided, such as reliance on tobacco, alcohol or other addictive substances. Parents can also use digital management software to restrict their children's phone usage to reduce the potential risks of communicating with anonymous or aggressive persons.

Expanding public knowledge on addiction is an important way to prevent addictive behaviours. Professionals should make use of various media, including published articles, animation, audio and broadcasts, to enhance the accessibility of informative content for different groups.

\section{Key population}

Groups with higher risks of addiction include persons with sleep disorders, smokers, drinkers, adolescents, individuals who are particularly susceptible to stress or have specific personality characteristics (eg, impulsiveness, curiosity), people who to date have abstained from addictive behaviours, patients with mental diseases and so on. ${ }^{11} 12$ According to the changes to addictive behaviours after previous public health or other major stress events, training related to adaptive coping skills should be available for high-risk groups. This includes maintaining healthy lifestyles, stress management, coping to a reasonable degree with negative emotions and seeking psychological assistance, all of which can serve to identify additional risk factors as early as possible and prevent addiction through further intervention.

Psychological intervention hotlines and outpatient services that screen for addictive behaviours should be widely available to improve early detection and intervention. In addition to the aforementioned high-risk groups, visitors should also be asked about the following problematic characteristics that may manifest during a pandemic: whether their dosages and frequency of substance use have increased compared with previous events; whether they have been able to quit again; whether they now rely on any addictive substances to relieve negative emotions; and 
whether they experienced withdrawal symptoms after reducing or quitting substance use. Patients who are known to have elevated risk or have the aforementioned characteristics should accept brief interventions or agree to further professional evaluations. Those who are diagnosed with substance use disorder should be transferred to specialised treatment institutions that provide addiction medicine to receive timely treatment.

\section{ADVICE FOR PATIENTS WITH ADDICTION PROBLEMS AND THEIR FAMILIES OR GUARDIANS}

Family support plays an important role in preventing relapses of patients with addiction problems. Family members can reduce patient cravings and prevent regression by stabilising family relationships while helping them reduce negative emotions and cope with high-risk factors. Patients with addiction problems and their families should pay special attention to all of the followings:

\section{Understand and pay close attention to emotions}

Patients and their families can learn to understand their emotional states using appropriate psychometric assessment tools. Furthermore, they can also receive training to adjust and relieve negative emotions through mindfulness, meditation, relaxation training and other methods. Patients and their families should realise that mild tension and anxiety during stressful situations are considered normal reactions that in turn can help them remain alert and mitigate their nervousness. Once uncontrolled negative emotions begin to have a significant impact on daily life and work, patients should request an online consultation or visit a professional institution for appropriate treatment.

\section{Build one's own social support system}

Talking with others can effectively help individuals relieve tension and anxiety. Therefore, the family members of patients with addiction problems can establish their own social support systems. Family members and close friends should communicate more frequently and adopt methods of 'healing in groups' to help each other solve problems encountered during a pandemic. If this method does not effectively solve the problem and bouts of severe insomnia, depression or anxiety continue to occur, the best advice is to request a phone consultation with a mental health institution. If necessary in severe cases, the person should visit a psychiatric emergency department to receive appropriate treatment.

\section{Proper management of addictive substances: strengthening observation} and support for patients

During a pandemic, negative emotions may increase substance abuse. Family members should carefully monitor all addictive substances at home, including alcohol and sedative hypnotics. For patients with drug addiction, family members should control all finances to ensure that the patient is unable to acquire drugs. After using addictive substances, patients with addiction problems often change their diets, sleep patterns and daily routines. Therefore, these often serve as indirect indicators that the patient has suffered a relapse simply by observing changes in their daily life.

\section{Proper responses to withdrawal symptoms and relapses}

Patients who are currently using addictive substances, those who have been using alcohol and sedative hypnotics for a long time, or patients who are receiving drug maintenance therapy may experience withdrawal symptoms as a result of the pandemic's impact. When this occurs, they should seek immediate help from professional institutions. When a patient relapses for the first time, family members should stabilise their emotions and avoid casting excessive blame on the patient. At the appropriate time, they can analyse the causes and discuss countermeasures together with the patient to avoid further recurrences. If the patient has had repeated relapses, they should be observed closely, advised patiently and escorted to a medical institution for treatment as soon as possible once the pandemic is under control or when the conditions are considered safe enough to visit public facilities. If the relapse has resulted in a physical or psychiatric emergency, the patient should be taken to a professional medical institution for emergency treatment.

\section{Actively acquire knowledge related to COVID-19 and strengthen self-protection} Patients and families can acquire scientific knowledge, learn the protective measures for COVID-19 from official media sources, and control the quality of information to avoid negative effects caused by false or excessive information. At the same time, social resources, including community portals, media outlets and hotlines, can be used to alleviate negative emotions and concerns.

\section{SUGGESTIONS FOR REHABILITATION TREATMENT CENTRES}

At present, China's intervention institutions for drug use and addiction include primarily compulsory isolation drug rehabilitation institutions, voluntary rehabilitation facilities, methadone maintenance treatment clinics and community treatment centres. During a pandemic, these institutions should take preventive measures against COVID-19 while ensuring the continuity of treatment and rehabilitation to the greatest possible extent. In response to practical difficulties, the various types of institutions should offer corresponding solutions whenever possible.

\section{Drug treatment and rehabilitation institutions}

Isolation and detoxification agencies of the public security and judicial systems are all stringently managed. Limitations to rehabilitation activities, family visits, communication and related problems may cause patients to experience emotional stress with corresponding physical symptoms during a pandemic. In severe cases, negative effects, self-injury or even psychotic symptoms may occur. At the same time, appropriate site management and services may be hindered. Possible actions to address these circumstances include the following: 
Patients can be sent outdoors (playground) or to a rehabilitation treatment room in groups, subject to safety regulations

Through reasonable planning and organisation, participant activity spaces can be amplified based on the premise that closed isolation may have been a root cause of their negative behaviour. In these spaces, appropriate recreation can be offered, such as yoga, mindfulness exercises, aerobics, t'ai chi, baduanjin and other sports that have been found to reduce anxiety, irritability and stress. For these rehabilitation activities, individuals should maintain social distancing of more than 1 metre, wear masks and pay attention to disinfection and ventilation in the activity areas.

\section{Enhance health education in rehabilitation institutes}

Posters, missionary videos and health education lectures can be used to educate patients about COVID-19 in a venue. This can help improve patients' ability to identify diseases, understand prevention and control techniques, and reduce the risk of negative emotions caused by inadequate knowledge or misunderstanding.

\section{Strengthen communication with family members by allowing family calls}

If possible, the venue should provide family telephones to allow patients to communicate via telephone or video so they are kept abreast of their situation and receive care and encouragement from family members, thereby alleviating potential negative emotions.

Receive help from specialist agencies when necessary

Most psychological problems suffered by patients can be resolved by using online consultation services provided by various medical institutions or other social services. However, for patients with more severe emotional disorders or persistent hallucinations, impulsive violence or other detrimental behaviours, appropriate medical treatment should be sought based on the evaluation of a professional physician. Commonly used medications include benzodiazepines, antidepressants, mood stabilisers or antipsychotics.

\section{Methadone maintenance treatment facilities}

For patients receiving methadone maintenance treatment, the dual effects of opioid addiction and pandemic stress may leave patients more prone to mental and psychological distress. In addition, certain circumstances will make treatment of such patients difficult, including isolation management, fear of being infected when going out to take medication, conscious avoidance of treatment and complications of the medication process under pandemic conditions. Open management and treatment in the community may also increase the risk of relapse. Possible actions to address these issues include the followings:

Provide a safer medical environment and alleviate patient concerns about infection during daily medication

Methadone maintenance treatment sites should manage the environment of the entire facility, maintain clean and ventilated spaces, disinfect regularly, and ensure that all medical personnel adhere to personal protective measures. Patients should be assisted when taking medicine in groups at clinics, and long line-ups should be avoided. Patients receiving medication should be required to wear a mask and cooperate during body temperature measurements. They should be questioned about any related clinical symptoms and their epidemiological history, such as cough, shortness of breath, fatigue, diarrhoea and so on. Those with any abnormalities should be reported to the appropriate departments for further treatment.

\section{Pay more attention to the education and prevention of COVID-19 and screening evaluations}

To reduce the risk of cross-infection, remote networks and psychological interventions by telephone can be implemented. If a patient's mental and behavioural problems have affected regular daily life and social functioning, the patient should be referred to a mental health institution for further diagnosis and treatment in a timely manner subject to local safety measures.

Maintain the stability of the treatment plan and prevent additional substance addiction During a pandemic, care should be taken before avoiding or adjusting any methadone dose to prevent withdrawal symptoms or fluctuating conditions that increase the difficulty of treatment. Combined with local methadone maintenance treatment policies and outpatient management regulations, to ensure safety, patients in stable condition with good compliance records can be considered for methadone take-home services or provided with alternative treatments such as Suboxone to minimise risks of cross-infection.

\section{Voluntary drug treatment agencies}

Voluntary detoxification is an important part of our drug rehabilitation system. At present, the domestic voluntary drug treatment institutions encompass primarily the addiction specialty departments of psychiatric hospitals and independent drug treatment hospitals. In addition to providing diagnoses and treatment services for drug dependence and related disorders, they also conduct outpatient and inpatient treatment of issues related to substance addiction, including alcohol and sedative hypnotics and behavioural addiction. During this COVID-19 pandemic, the normal diagnoses and treatment of patients with substance and behavioural dependence throughout the community are inevitably affected.

\section{Strengthen departmental coordination and optimise the treatment model}

The goal should be to establish sound coordination mechanisms with public security agencies, comprehensive medical institutions, community drug treatment and rehabilitation agencies, drug maintenance and treatment agencies, and other addiction-related units. Also important is the fortification of contacts and liaisons with consultants. Given the actual situation in any local area, it is necessary to refine the work content and procedures to further 
ensure that persons with substance abuse and behavioural problems can receive prompt medical treatment, timely referrals and hospitalisation when necessary.

\section{Strengthen publicity and expand the coverage of treatment and prevention of addictive diseases}

Voluntary detoxification agencies should not only ensure routine diagnoses and treatment but also assume the responsibility of preventing addictive behaviours caused by serious public health events. Measures like science popularisation and professional training to assist the general population's ability to improve selfscreening for substance abuse and behavioural problems also benefit the community medical institutions and non-addiction-related professional medical personnel in their attempts to identify addiction-related disorders and to expand the coverage of prevention and treatment services. The screening of addictive substance use, prevention and education for clinic patients, as well as the timely identification, diagnosis and treatment of related mental and behavioural problems may all occur under stressful conditions. Follow-up sessions can be conducted through appropriate communication channels (eg, phone, email, WeChat and so on).

\section{Strengthen internal management to ensure treatment effectiveness}

It is necessary to improve the management of facilities and all hospitalised patients, to adopt appropriate measures to isolate and prevent infection from spreading among both the hospital staff and patients, and to eliminate the introduction of possible sources of out-of-hospital external infections. Also important is the dissemination of related medical knowledge and the provision of pharmaceutical and/or psychological interventions to treat mental and psychological problems such as stress, negative feelings and insomnia. Also, the optimisation of outpatient appointment schedules would enable patients to visit doctors in an orderly manner and prevent infection risks caused by too many persons gathering in confined spaces. Telemedicine services can also be provided to discharged patients by telephone, online consultation and other methods to enhance continuous service quality.

\section{MANAGEMENT OF COMORBID COVID-19 AND/OR OTHER DISEASES IN PATIENTS WITH ADDICTION PROBLEMS}

Patients with addiction problems often suffer from several physical and mental illnesses. This substantially increases the difficulty of treatment once a patient contracts COVID-19. The treatment of comorbid physical and mental illnesses often requires the deployment of alternative addiction treatments.

\section{Suspected or actual COVID-19 infection} in patients with addiction problems

Patients with addiction problems who are either suspected of being infected or are infected with COVID-19 should be isolated immediately for a predetermined period. Isolation, treatment and recovery management should be provided in accordance with the stipulated requirements for suspected or infected patients. Special attention should be paid when asking patients about previous and current substance abuse, withdrawal symptoms previously experienced, and other physical diseases. Addiction professionals should be invited to join treatment groups, thereby allowing general physicians and these specialists to jointly formulate the most appropriate treatment plans. When controlling physical diseases, medical teams should treat the symptoms generated by withdrawal from addictive substances. Patients suffering from the dual effects of COVID-19 and withdrawal may complicate the disease and increase the difficulty of treatment. Therefore, the patient's vital signs should be closely monitored and variances should be treated promptly. Psychosocial interventions should also be provided during treatment.

Other comorbid physical or mental diseases in patients with addiction problems

Often that patients with addiction problems have other comorbid physical or mental diseases. Reports show that about $50 \%-70 \%$ of patients with addiction problems suffer from other mental illnesses, and 20\%-50\% of patients with other mental illnesses also suffer from substance use disorders. Also, long-term use of psychoactive substances may lead to malnutrition, low immune function and a higher risk of contracting other physical diseases. ${ }^{13}$ Practical difficulties during this pandemic have inconvenienced patients from receiving timely medical treatment, and this in turn may lead to increased risk of addictive substance use. Therefore, more attention should be paid to the physical and mental conditions of patients with comorbid conditions. If treatments not involving drugs are ineffective in coping with negative emotions and/or exacerbations of physical illnesses, patients should be instructed to seek professional treatment as soon as possible.

In sum, according to the theory of stress-facilitated addictive behaviours when coupled with environmental and other restrictions, the prevalence of patients with addiction-related disorders may increase during a pandemic, while existing patients may exacerbate their poor condition or even relapse, ${ }^{14}$ thus posing greater challenges for the healthcare personnel. This article provides a summary of the common addictive behaviour problems that may occur or worsen among the general population and patients with addiction problems during a pandemic and proposes possible intervention methods. We hope to use this research as a foundation to further explore a set of effective prevention and treatment methods for addiction that are bound to be necessary during future major public health crises in order to prepare and benefit the general population.

\footnotetext{
Author affiliations

${ }^{1}$ Drug Abuse Treatment Department, Shanghai Mental Health Center, Shanghai, China

${ }^{2}$ Drug Abuse Treatment Department, Affiliated Brain Hospital of Guangzhou Medical University, Guangzhou, Guangdong, China

${ }^{3}$ Shanghai Mental Health Center, Shanghai, China
} 
${ }^{4}$ Mental Health Institute, Second Xiangya Hospital, Central South University, Changsha, Hunan, China

${ }^{5}$ Mental Health Institute, Second Xiangya Hospital,

Changsha, Hunan, China

${ }^{6}$ Peking University Sixth Hospital, Beijing, China

${ }^{7}$ Peking University School of Basic Medical Sciences,

Beijing, China

${ }^{8}$ The Affiliated Brain Hospital, Guangzhou Medical

University, Guangzhou, Guangdong, China

${ }^{9}$ Mental Health Institute, Sichuan University West China

Hospital, Chengdu, Sichuan, China

${ }^{10}$ Drug Abuse Treatment Department, Beijing

Huilongguan Hospital, Beijing, China

${ }^{11}$ Drug Abuse Treatment Department, Beijing An Ding

Hospital, Beijing, China

${ }^{12}$ Drug Abuse Treatment Department, Wuhan Mental

Health Center, Wuhan, Hubei, China

${ }^{13}$ Drug Abuse Treatment Department, Brain Hospital of

Hunan Province, Changsha, China

${ }^{14}$ Huzhou Third People's Hospital, Huzhou, Zhejiang,

China

${ }^{15}$ Drug Abuse Treatment Department, Ningbo University

Medical School, Ningbo, Zhejiang, China

${ }^{16}$ Drug Abuse Treatment Department, Xinxiang Medical

University Affiliated Second Hospital, Xinxiang, Henan,

China

${ }^{17}$ Xinjiang Medical University Affiliated First Hospital,

Urumqi, Xinjiang, China

${ }^{18}$ Mental Health Department, First Affiliated Hospital of

Harbin Medical University, Harbin, Heilongjiang, China

${ }^{19}$ Mental Health Department, Shandong Province

Ankang Hospital, Jining, Shandong, China

${ }^{20}$ Huayou Health Care Center, Beijing, China

${ }^{21}$ Drug Abuse Treatment Department, Shenzhen

Kangning Hospital, Shenzhen, Guangdong, China

${ }^{22}$ Drug Abuse Treatment Department, Shandong Mental

Health Center, Jinan, China

Contributors JD and NF drafted the manuscript. WH, TL and MZ revised the final manuscript. All the following authors reviewed and approved the manuscript: MZ, JD, HJ, NZ, WH, HC, TL, XX, XW, LL, HS, BL, JS, Y-PN, NF, XLa, SX, JL, WG, YN, LS, YL, XLi, $X Z, M Q, W Z, R Z, H H, Y X, Z S, R Z, M Y, F L$ and WY.

Funding This work was supported by the Program of Shanghai Academic Research Leader (17XD1403300), the Shanghai Key Laboratory of Psychotic Disorders (13DZ2260500), the Shanghai Intelligent Engineering Technology Research Center for Addiction and Rehabilitation (19DZ2255200), and the
Shanghai Clinical Research Center for Mental Health (19MC1911100).

Competing interests None declared.

Patient consent for publication Not required.

Provenance and peer review Not commissioned; externally peer reviewed.$$
\text { (2) }
$$$$
\text { OPEN ACCESS }
$$

Open access This is an open access article distributed in accordance with the Creative Commons Attribution Non Commercial (CC BY-NC 4.0) license, which permits others to distribute, remix, adapt, build upon this work non-commercially, and license their derivative works on different terms, provided the original work is properly cited, appropriate credit is given, any changes made indicated, and the use is non-commercial. See: http://creativecommons.org/licenses/by-nc/4.0/.

(c) Author(s) (or their employer(s)) 2020. Re-use permitted under CC BY-NC. No commercial re-use. See rights and permissions. Published by BMJ.

JD and TL are joint first authors.

\section{Check for updates}

To cite Du J, Fan N, Zhao M, et al. General Psychiatry Epub ahead of print: [please include Day Month Year]. doi:10.1136/gpsych-2020-100252

Received 23 April 2020

Revised 30 May 2020

Accepted 4 June 2020

General Psychiatry 2020;33:e100252.

doi:10.1136/gpsych-2020-100252

ORCID iD

Min Zhao http://orcid.org/0000-0002-4551-043X

\section{REFERENCES}

1 Lin L, Cun SY. Psychiatry [M]. 6th edn. Beijing: People's Health Press, 2018.
2 COVID-19 pneumonia joint prevention and control mechanism group of the State Council. The notice on strengthening management and treatment of severe mental disorders during the period of COVID-19 pneumonia [EB/OL]. (2020-0218). Available: http://www.gov.cn/xinwen/ 2020-02/19/content_5480748.htm

3 COVID-19 pneumonia joint prevention and control mechanism group. "[EB/OL]." (2020-02-25) on the scientific and accurate basis for the prevention and control of COVID-19 pneumonia epidemic situation in accordance with the law. Available: http:// www.gov.cn:8080/zhengce/2020-02/25/ content_5483033.htm

4 Qiu J, Shen B, Zhao M, et al. A nationwide survey of psychological distress among Chinese people in the COVID-19 epidemic: implications and policy recommendations. Gen Psychiatr 2020;33:e100213.

5 Vlahov D, Galea S, Resnick H, et al Increased use of cigarettes, alcohol, and marijuana among Manhattan, New York, residents after the September 11th terrorist attacks. Am J Epidemiol 2002;155:988-96.

6 Moise IK, Ruiz MO. Hospitalizations for substance abuse disorders before and after Hurricane Katrina: spatial clustering and Area-Level predictors, new Orleans, 2004 and 2008. Prev Chronic Dis 2016;13:E145.

7 Xiaolu R, Wenwen W, Ali R, et al. Feasibility of studying a brief intervention to help Chinese villagers with problem alcohol use after an earthquake. Alcohol Alcohol 2017;52:472-6.

8 Reed P, Vile R, Osborne LA, et al. Problematic Internet usage and immune function. PLoS One 2015;10:e0134538.

9 Keepers GA. Pathological preoccupation with video games. J Am Acad Child Adolesc Psychiatry 1990;29:49-50.

10 West China Medical University. New coronavirus handbook for public psychological protection [M]. West China Medical University, 2020.

11 Wemm SE, Sinha R. Drug-Induced stress responses and addiction risk and relapse. Neurobiol Stress 2019;10:100148.

12 Thakkar MM, Sharma R, Sahota P. Alcohol disrupts sleep homeostasis. Alcohol 2015;49:299-310.

13 Straub RH, Cutolo $\mathrm{M}$. Psychoneuroimmunology-developments in stress research. Wien Med Wochenschr 2018:168:76-84.

14 Bao Y, Sun Y, Meng S, et al. 2019-nCoV epidemic: address mental health care to empower Society. Lancet 2020;395:e37-8.

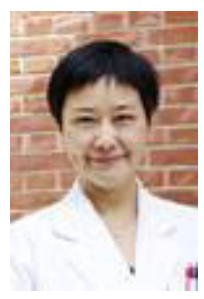

Dr. Jiang Du received her doctor's degree from Shanghai fiao Tong University School of Medicine. She is currently the director of Drug Abuse Treatment Center at Shanghai Mental Health Center. Her research interests include drug abuse prevention and intervention. 